This item was submitted to Loughborough's Research Repository by the author.

Items in Figshare are protected by copyright, with all rights reserved, unless otherwise indicated.

\title{
A probabilistic method for calculating the usefulness of a store with finite energy capacity for smoothing electricity generation from wind and solar power
}

\section{PLEASE CITE THE PUBLISHED VERSION}

http://www.ipss.org.uk/

\section{PUBLISHER}

Elsevier / International Power Sources Symposium (IPSS)

VERSION

AM (Accepted Manuscript)

\section{LICENCE}

CC BY-NC-ND 4.0

\section{REPOSITORY RECORD}

Barton, John P., and David Infield. 2019. "A Probabilistic Method for Calculating the Usefulness of a Store with Finite Energy Capacity for Smoothing Electricity Generation from Wind and Solar Power". figshare. https://hdl.handle.net/2134/14438. 
This item was submitted to Loughborough's Institutional Repository (https://dspace.lboro.ac.uk/) by the author and is made available under the following Creative Commons Licence conditions.

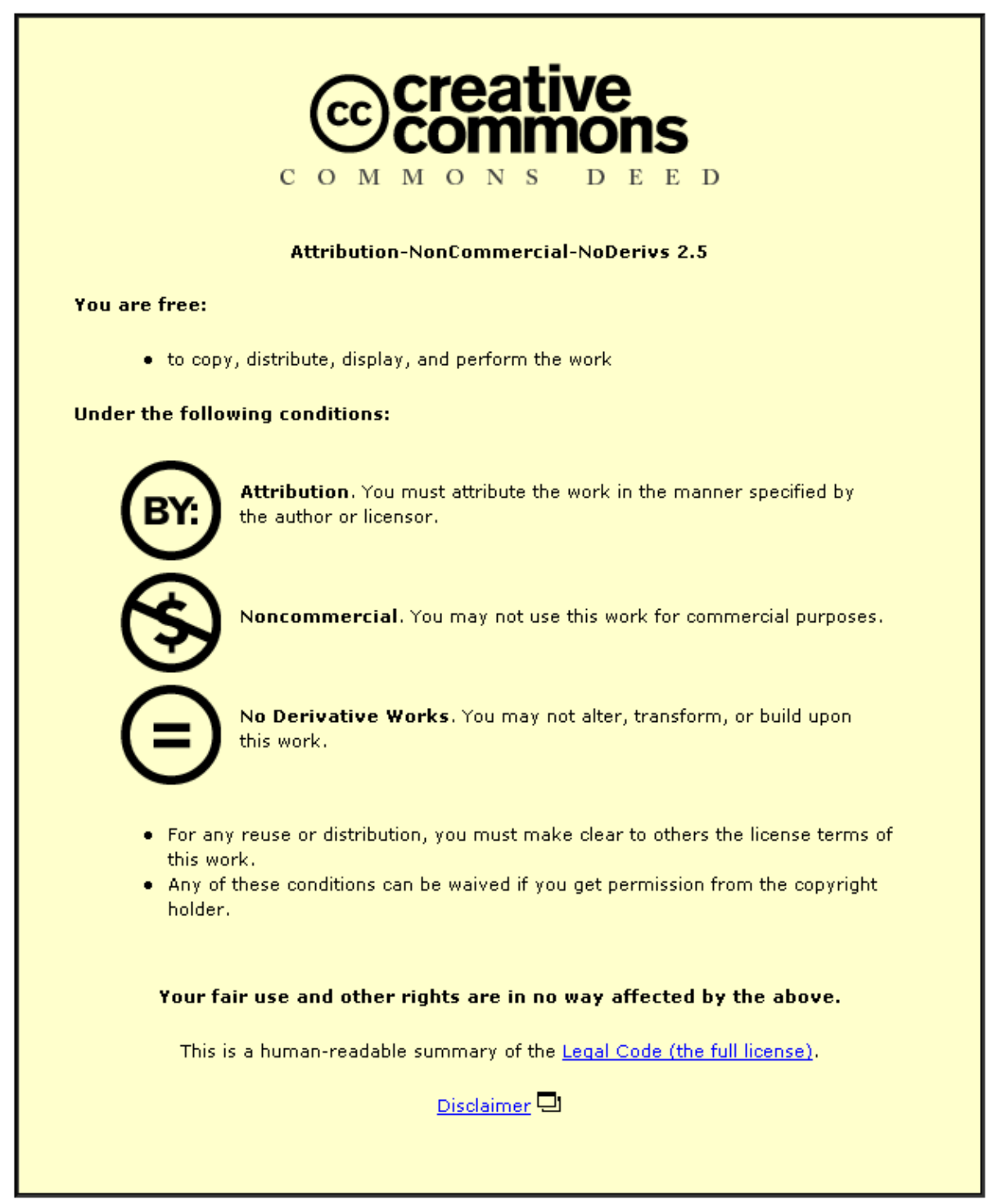

For the full text of this licence, please go to: http://creativecommons.org/licenses/by-nc-nd/2.5/ 


\title{
A Probabilistic Method for Calculating the Usefulness of a Store with Finite Energy Capacity for Smoothing Electricity Generation from Wind and Solar Power
}

John P. Barton ${ }^{*}$ and David G. Infield

CREST, Department of Electronic and Electrical Engineering, Loughborough University, Leicestershire, UK, LE11 3TU

\begin{abstract}
This paper describes a novel method of modelling an energy store used to match the power output from a wind turbine and a solar PV array to a varying electrical load. The model estimates the fraction of time that an energy store spends full or empty. It can also estimate the power curtailed when the store is full and the unsatisfied demand when the store is empty. The new modelling method has been validated against timestepping methods and shows generally good agreement over a wide range of store power ratings, store efficiencies, wind turbine capacities and solar PV capacities. Example results are presented for a system with 1MW of wind power capacity, 2MW of photovoltaic capacity, an energy store of 75\% efficiency and a range of loads from 0 to $3 \mathrm{MW}$ average.
\end{abstract}

Author Keywords: Energy storage, Stand-Alone Power Systems, Modelling, Photovoltaics, Voltage control, Wind power generation

\section{Introduction}

Wind power is currently the fastest growing renewable energy source worldwide

\footnotetext{
* Corresponding author. Tel: 44 (0)1509 228140; email J.P.Barton@lboro.ac.uk
} 
with photovoltaics (PV) running a close second, albeit from a lower base. Wind capacity, and to a lesser extent generation from PV pose challenges for grid connection and network operation. Wind turbine output depends directly on wind speed and varies across a wide range of time scales. PV output is variable but to a greater extent predictable.

The computational method developed relies upon spectral description of the wind speed and solar radiation together with a standard power curve for the wind turbines and well-defined PV array characteristics. The electricity load, based on data from Leicester, is calculated as a function of hour of the day, type of day (working day or non-working day) and month of year. An example for a weekday in December is shown in Fig. 1, where it is compared with the National Grid typical winter demand profile measured on $3^{\text {rd }}$ December 2002 and approximately scaled to the same average [1].

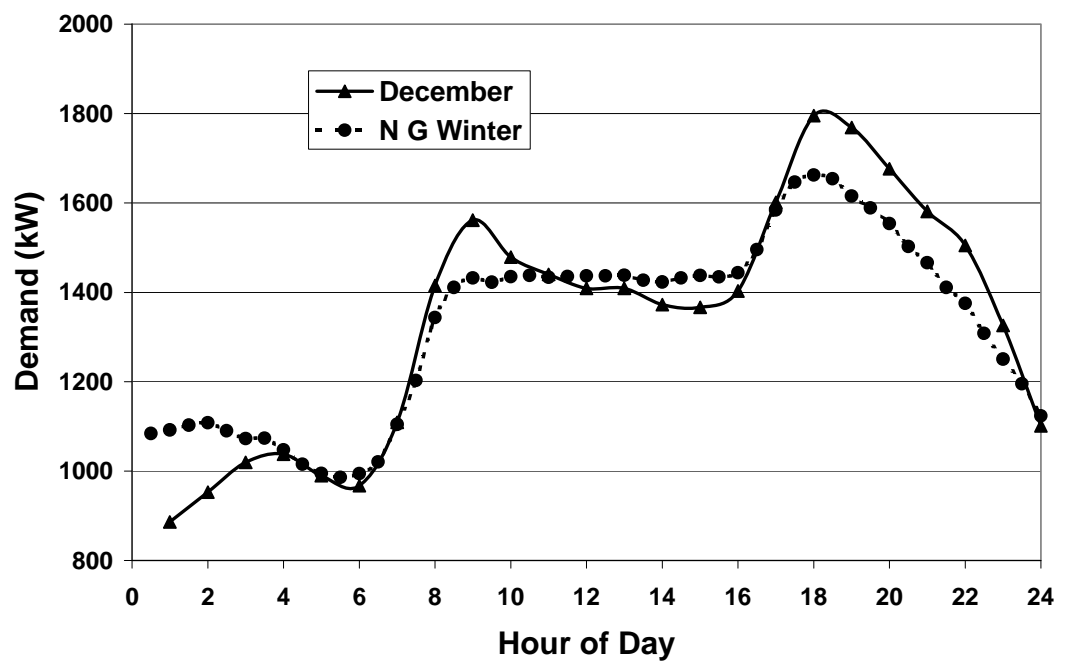

Fig. 1. December weekday electricity load profiles 
Understandably, the Leicester load data exhibits greater peaks in the morning and evening than the national average profile, reflecting reduced smoothing due to diversity and possibly a greater proportion of domestic consumers. Nevertheless, the profiles are similar.

Conventional electricity systems can accommodate at least $5 \%$ of wind powered generation, and probably more, with few adaptations [2]. The variability of small amounts of wind or solar power can be accommodated in the same way as variations in electrical load: by varying the electrical generation from fossil fuel generators. However, if renewable sources are to play their part in a $60 \%$ reduction in carbon dioxide emissions, the fraction of intermittent renewable generation will increase well above $5 \%$. This will require a radical new approach to electrical power system management and a potentially important role for energy storage.

In the approach presented here the energy storage system is represented as a series of filters in the spectral domain applied to the solar and wind variation spectra.

\section{System Modelling}

The energy rating of a store increases with the time-scale of charging and discharging cycles [3]. Energy storage options always become more expensive as the time scale (a typical charge-discharge cycle) of that storage increases. A cost-effective system may therefore have a small store size in energy capacity terms, but with a relatively large renewable energy power surplus and hence storage power rating (at least as far as charging goes). Only a mathematical model of the system can determine the optimal design with any confidence.

Energy systems including wind power, solar power, loads and energy storage can be extremely complex to model. Wind speed, solar irradiance and loads all vary on all 
time scales from seconds to years. The state-of-charge of an energy store depends on the history of energy supply and demand and its own operating characteristics.

Earlier work [3] developed a novel method of calculating energy flows to and from an energy store, but made only a crude attempt to size the store.

This paper extends the methodology presented in [3] to include an improved calculation of store size and a more sophisticated spectral analysis of the intermittency of renewable sources. The method simulates electrical power systems with large fractions of intermittent renewable generation and energy storage. It does not require time series of weather data. Instead it uses spectral analysis of time series de-trended to account for deterministic cyclic variations (seasonal and diurnal) together with relevant probability distributions,

Some loss of accuracy may be apparent because of the necessary simplifying assumptions, but it is expected that the probabilistic method will provide a fast and practical tool for feasibility studies, early system design, and investment decisions.

Time-step simulation remains useful however, as a check that the design will perform as intended, to fine-tune the design, and to devise the control software of the energy management system.

In this paper, the method has been developed for storage and delivery of energy over a period of 24 hours, reflecting the diurnal nature of wind, solar and load variations. The approach can straightforwardly be extended to other time scales, for example an hour or a week, and this is the subject of ongoing research.

\subsection{Wind Turbine Modelling}

A generic power curve for a $1 \mathrm{MW}$ wind turbine has been used for the calculations presented here. This curve, Fig. 2, was constructed from data for commercially 
available wind turbines [4] and was previously used in [3] and [5]. The cut in speed is $3 \mathrm{~m} \mathrm{~s}^{-1}$ and the rated wind speed at $13 \mathrm{~ms}^{-1}$ is typical of large modern turbines.

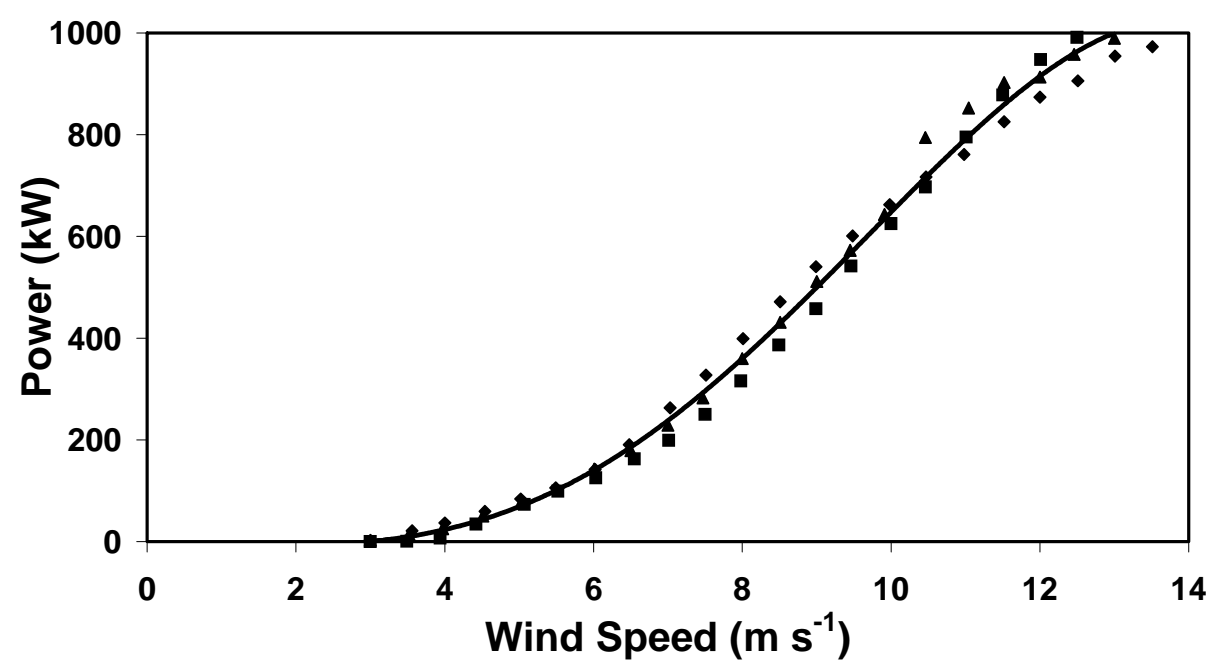

Fig. 2. Composite 1MW turbine power curve from three commercial turbines 


\subsection{Wind Speed Variations}

The Van Der Hoven spectrum [6] describes wind speed variations up to 1000 hours in duration. Corresponding spectra must be calculated from local wind speed data in order to predict accurately the character of wind power variations at a given location. Data has been analysed for a well-documented location, The Rutherford Appleton Laboratory (RAL) in Oxfordshire, UK,

Fig. 3.

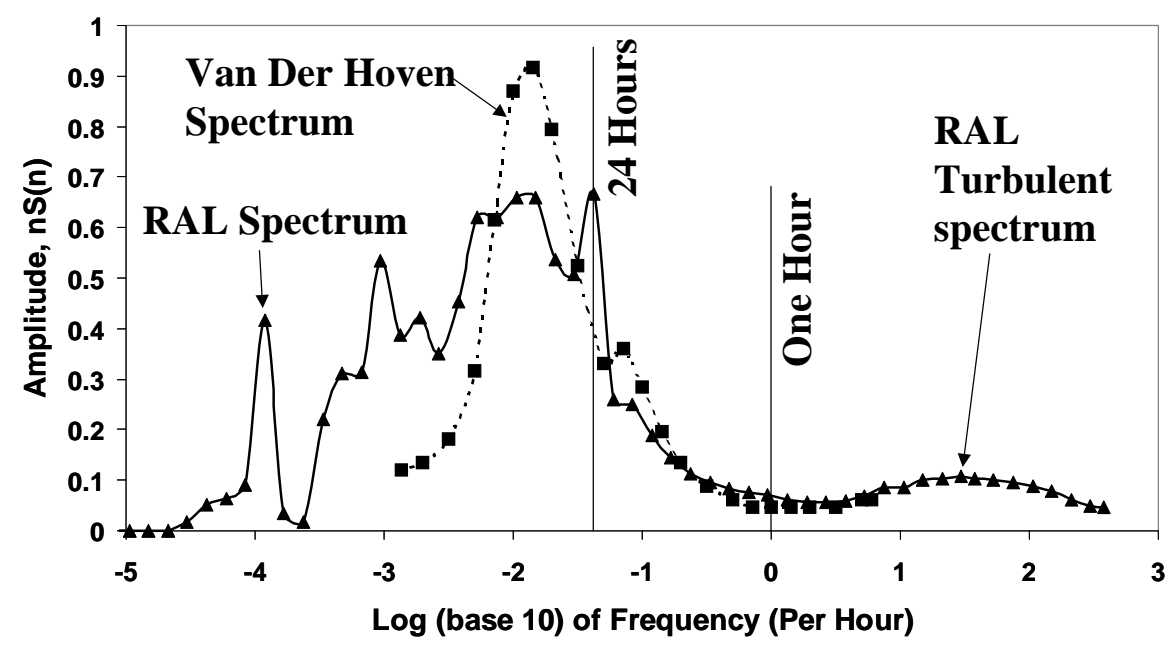

Fig. 3. Wind speed variation spectra adjusted to $8 \mathrm{~m} / \mathrm{s}$ mean wind speed

\subsection{Solar Irradiance Variations}

A spectrum has been calculated in a similar way for solar irradiance, Fig. 4. Again, data from RAL has been used; the spectrum will be broadly applicable to central England. 


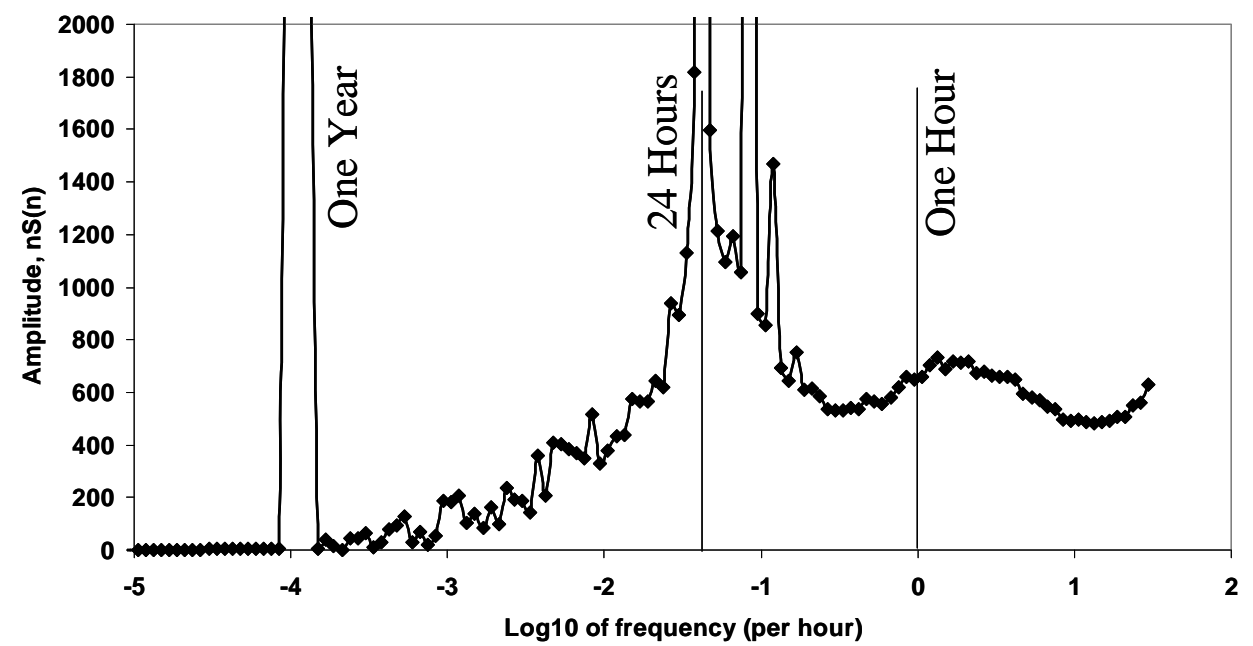

Fig. 4. Solar irradiance variation spectrum

This spectrum for solar variation (not to be confused with the solar electromagnetic spectrum) is dominated by peaks reflecting seasonal variation, diurnal variation and harmonics of the diurnal variation. These peaks are so large that they are not shown in Fig. 4 in order to reveal the broad remaining structure. The broadband spectrum from periods of months down to minutes represents a significant random variation in solar irradiance as big in percentage terms as that of the wind spectrum over the same range of frequencies. The broad hump of variation to the right of the diurnal peak represents variation due to passing clouds, while the tapered spectrum to the left of the diurnal peak represents weather systems.

\subsection{Solar Power Modelling}

The solar power output in $\mathrm{kW}$ was assumed to be equal to the global horizontal irradiance in $\mathrm{kW} \mathrm{m}^{-2}$ multiplied by the peak PV efficiency and the array area. In a real system, adjustments should be made for the inclination and azimuthal angles of the PV panel; the effect of beam radiation should be considered separately from that of diffuse radiation; temperature and spectral (wavelength) effects should be taken into 
account; the characteristics of a maximum power point tracker and power converter should also be included. However, all these effects are of secondary magnitude and outside the scope of this paper. The method presented here would be suitable for a feasibility study where the approximate results are sufficient for system sizing.

\subsection{Energy Store Modelling}

The charging and discharging power ratings of the store are unrestricted for the purposes of this model. The energy rating of the store, and its behaviour are calculated using the equations given in [5] and repeated below.

Filter functions have been applied to the spectra of wind speed variations of Fig. 3, and of solar irradiance variations as in Fig. 4, in order to calculate the probability distributions of period-average wind speed and irradiance, the distributions of wind speed and irradiance within each period, and the size (energy capacity) of the required store. For a given spectral frequency component, $\omega_{i}$ with amplitude, $A$ and phase angle $\varphi_{i}$, the instantaneous component is: $U_{i}=A_{i} \sin \left(\omega_{i} t+\phi_{i}\right)$. Averaging this quantity over the store period, $T$ gives the contribution from this frequency to the period-average wind speed or irradiance:

$$
\overline{U_{i}}=\frac{A_{i}}{\omega_{i} T}\left[\cos \phi_{i}-\cos \left(\omega_{i} T+\phi_{i}\right)\right] .
$$

This average is then squared and integrated over all $\varphi_{i}$ from 0 to $2 \pi$ to give the contribution to the variance in the period average wind speed or irradiance. The resulting integral is:

$$
V_{1 i}=\left(\frac{A_{i}}{\omega_{i} T}\right)^{2}\left[1-\cos \left(\omega_{i} T\right)\right] .
$$


This formula is the same as the low pass filter used by Infield [7] for storage modelling, but without the 2.4 empirical scaling factor. The filter for a 24-hour store is shown Fig. 5. Low frequency components (small $\omega$ ) have a relatively large effect on the period average wind speed or irradiance whereas high frequency components (large $\omega$ ) have a small effect on the period average. Low frequency components remain nearly constant throughout a time period, $T$. High frequency components of wind or solar variation complete many cycles during the time, $T$, and time spent above the long-term mean is approximately balanced by time spent below.

The variance of wind speeds or irradiance within a period, $T$ is calculated in a similar way, but this time, the important quantity is the difference between the instantaneous value, $U_{i}$ and the period-average, $\overline{U_{i}}$ :

$U_{i}-\overline{U_{i}}=A_{i} \sin \left(\omega_{i} t+\phi_{i}\right)-\frac{A_{i}}{\omega_{i} T}\left[\cos \phi_{i}-\cos \left(\omega_{i} T+\phi_{i}\right)\right]$ If this quantity is squared, integrated over time, $T$ and averaged over all possible values of $\varphi_{i}$, then the component of variance within period $T$ results:

$$
V_{2 i}=\frac{A_{i}^{2}}{2}-\left(\frac{A_{i}}{\omega_{i} T}\right)^{2}\left[1-\cos \left(\omega_{i} T\right)\right]
$$

This integral represents a high pass filter, previously derived by Bossanyi [8] and also shown in Fig. 5. It is actually the complement of the low pass filter function in the sense that summing the two time series resulting from application of the two filters to a given time series results in the original series.

A third filter function is used to calculate the variance in state-of-charge of a store associated with a particular frequency component, $\omega_{i}$. The accumulated energy added to or subtracted from a store during period, $T$ is the calculated from the integral of the difference between the instantaneous value, $U_{i}$ and the period average, $\overline{U_{i}}$. But this 
time, the instantaneous accumulated energy is squared and integrated again with respect to both time and phase angle to give the average variance in the excursion of state-of-charge from its value at the start of period, $T$ :

$$
V_{\text {SOC }}=\frac{A_{i}^{2}}{\omega_{i}^{2}}\left\{\frac{5}{6}+\frac{1}{6} \cos \left(\omega_{i} T\right)+\frac{2}{\omega_{i}^{2} T^{2}}\left[\cos \left(\omega_{i} T\right)-1\right]\right\}
$$

This third equation is applied to the original spectral density functions. Integration of the resulting filtered spectrum gives the variance of wind speed $\mathrm{x}$ time or irradiance $\mathrm{x}$ time. Low frequency components have little effect on the store, since their magnitude varies so little during period, $T$. High frequency components also have little effect on the store, since they complete many cycles during period, $T$, so each cycle accumulates and discharges very little energy. Only frequency components close to the period of the store have a significant effect on the state-of-charge, Fig. 5.

The wind speed $\mathrm{x}$ time is square-rooted and multiplied by the average gradient from the turbine power curve to produce a standard deviation of state-of-charge due to wind speed variation. The irradiance $\mathrm{x}$ time is square-rooted and multiplied by the solar PV peak capacity to produce another standard deviation of state-of-charge due to solar irradiance variations. A third, diurnal standard deviation of state-of-charge results from the average daily profiles of load, wind speed and solar irradiance. All three components of state-of-charge standard deviation are added in quadrature to give the total standard deviation in state-of-charge.

The effective energy capacity of the store is approximately given by twice the standard deviation of state-of-charge. The factor of 2 is empirical (rather like the factor of 2.4 used by Infield) but is intuitively the result of the symmetry about the mean value mentioned above. 


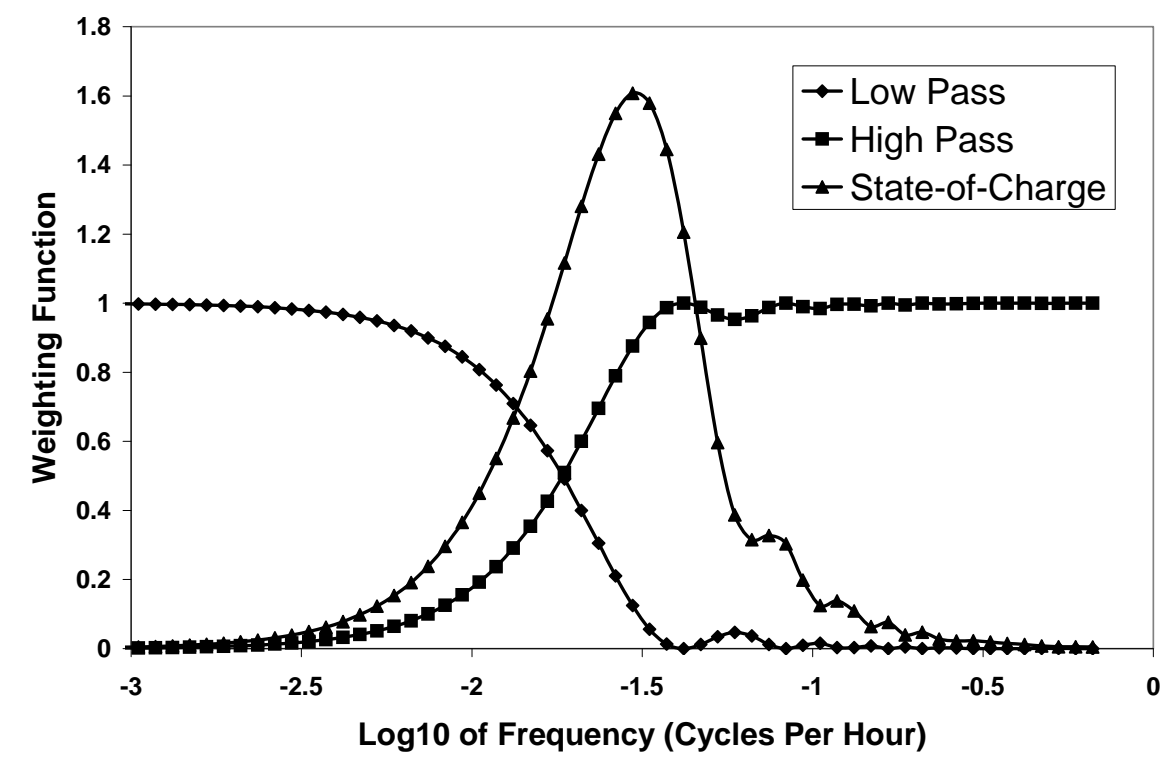

Fig. 5. Spectrum filter functions for a 24-hour store

\section{Model Validation}

All the cases modelled using the probabilistic methods have been validated by a time-step method with the same numerical inputs. The time-step method uses the same wind speed time series and solar irradiance time series from which the variation spectra were calculated. The time step method also uses the same time series of load data from which the daily load profiles were prepared.

All wind speed distributions have been corrected to a long-term mean wind speed of $8 \mathrm{~m} / \mathrm{s}$. The model is applied here to a storage time scale of 24 hours. This means that variations in power flows within one day are accommodated by the store, whereas longer-term variations are not. Stored energy cannot be transferred from one day to the next. 


\section{Example Results}

The results presented here are for a stand-alone electricity system supplying a time-varying load typical of a single $11 \mathrm{kV}$ feeder in a UK Midlands town. The assumed electricity system comprises 1MW of wind power capacity, 2MW of solar PV capacity and an energy store of 75\% efficiency capable of smoothing out energy surpluses and deficits over a maximum period of 24 hours.

The novel probabilistic method predicts the fraction of time that the store spends empty, full, emptying or filling. It also predicts the energy lost when the store is full, the unsatisfied demand when the store is empty, and the energy capacity of the store required. To illustrate the versatility of the probabilistic method, the load has been scaled between 0 and 3.25MW, and the results plotted in the following graphs. A low load represents an over-supply of renewable energy and a high load represents an under-supply of renewable energy.

The energy capacity of the store is calculated on days in which average supply is equal to, or closest to the average load (with an adjustment for the efficiency of the store), as these are the days when the store does the most 'work' of emptying and filling. A small load, for example $0.25 \mathrm{MW}$ is matched by renewable energy on relatively calm or dull days and requires only a small store, Fig. 6. Conversely, a large load, for example $1.75 \mathrm{MW}$, is only matched by renewable energy on very windy and sunny days. These are also the days in which wind power and solar power are most variable. Therefore, a large load requires a large store size, Fig. 6. If the average load is greater than $2 \mathrm{MW}$, it is never matched by renewable energy, and the store size is again calculated on the most windy and sunny days, when renewable energy is greatest and therefore comes closest to satisfying the load. Since the wind and solar power variations have greater percentage variability than the load, and since the solar 
and wind power variances are already at their maximum values, the store size does not significantly increase, if at all, with increasing load, Fig. 6.

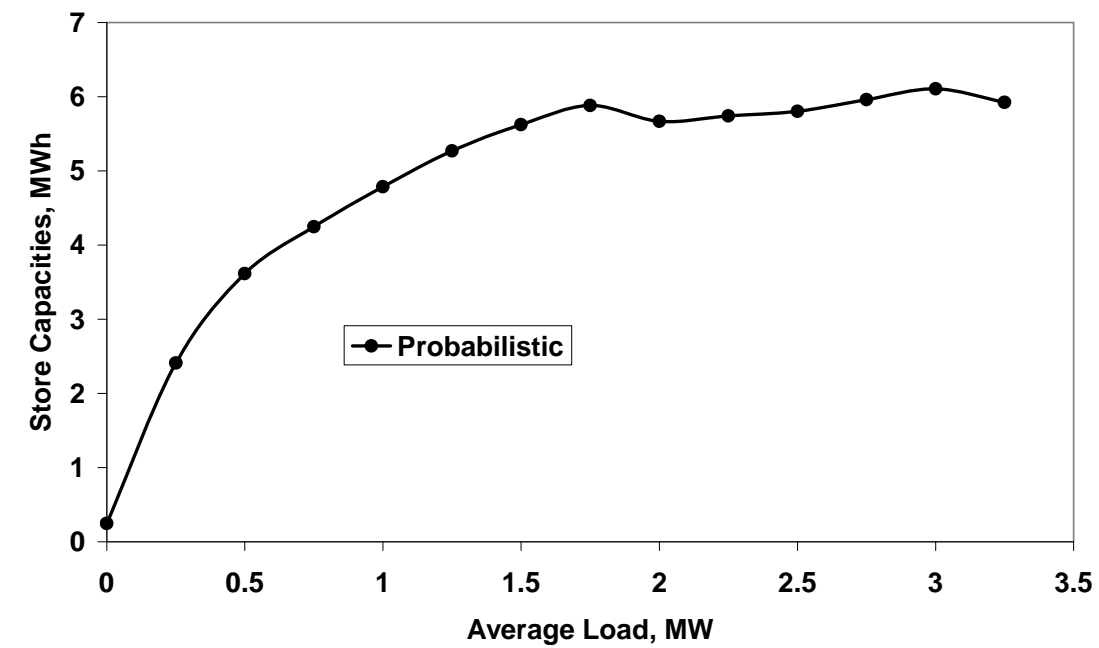

Fig. 6 Calculated store sizes for 24-hour storage periods

The probabilistic method predicts the excess load, the curtailed power and power flows to and from the store very well, as validated by time-step methods, Figs. 7 to 9: 


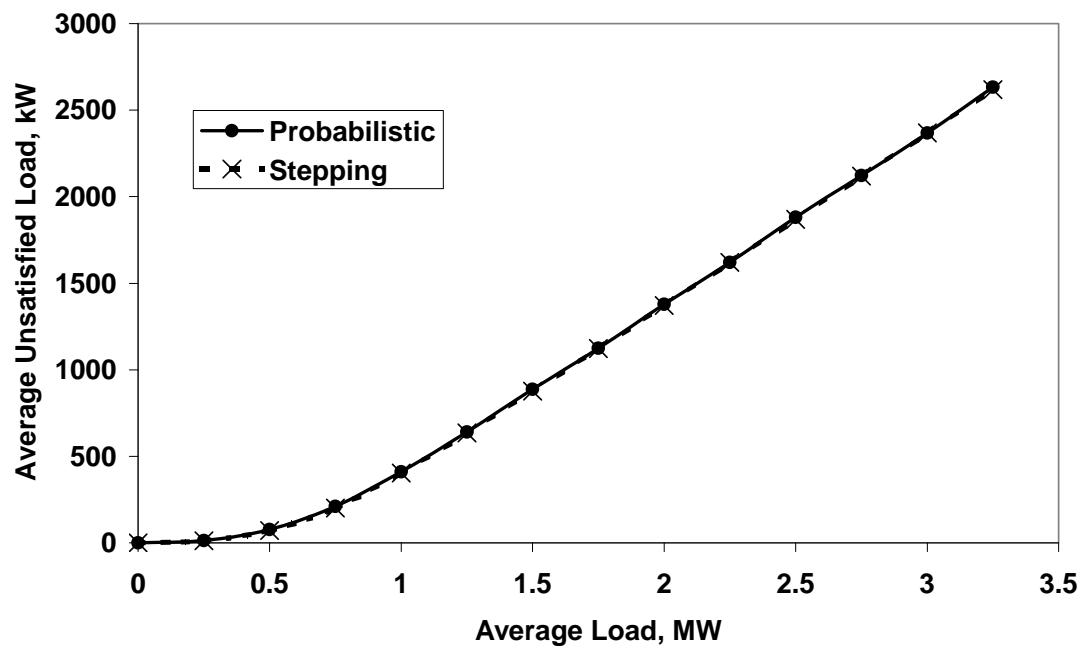

Fig. 7. Load unsatisfied by renewable energy due to the store being empty

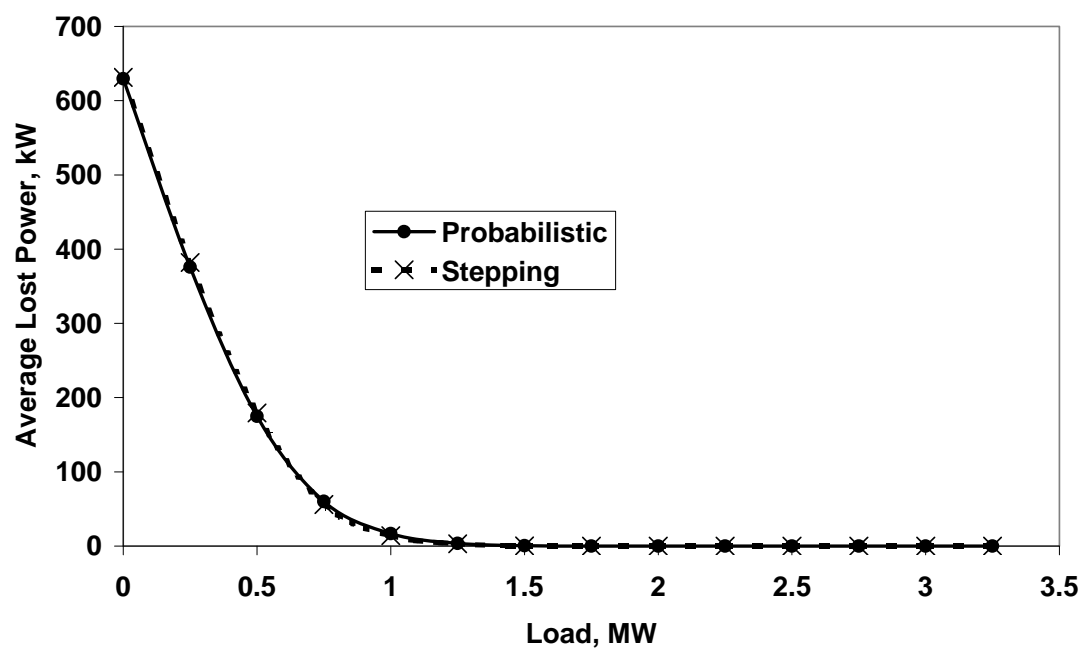

Fig. 8. Renewable power curtailed due to the store being full 


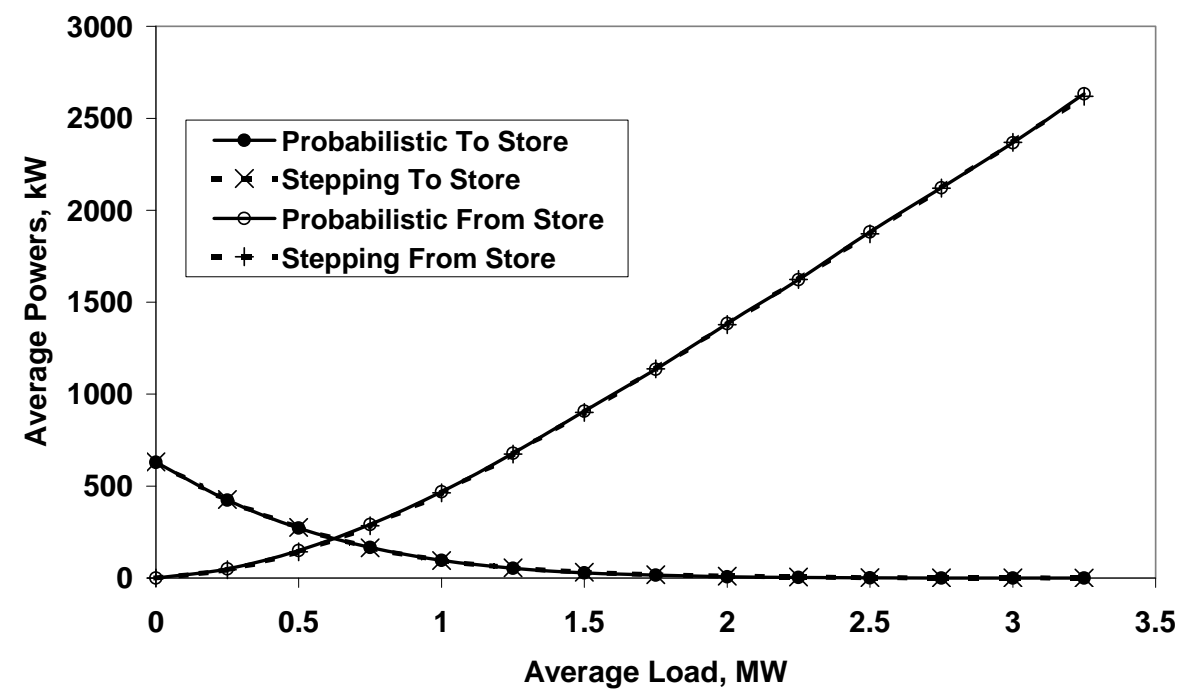

Fig. 9. Average power flows to and from the store (neglecting full and empty effects)

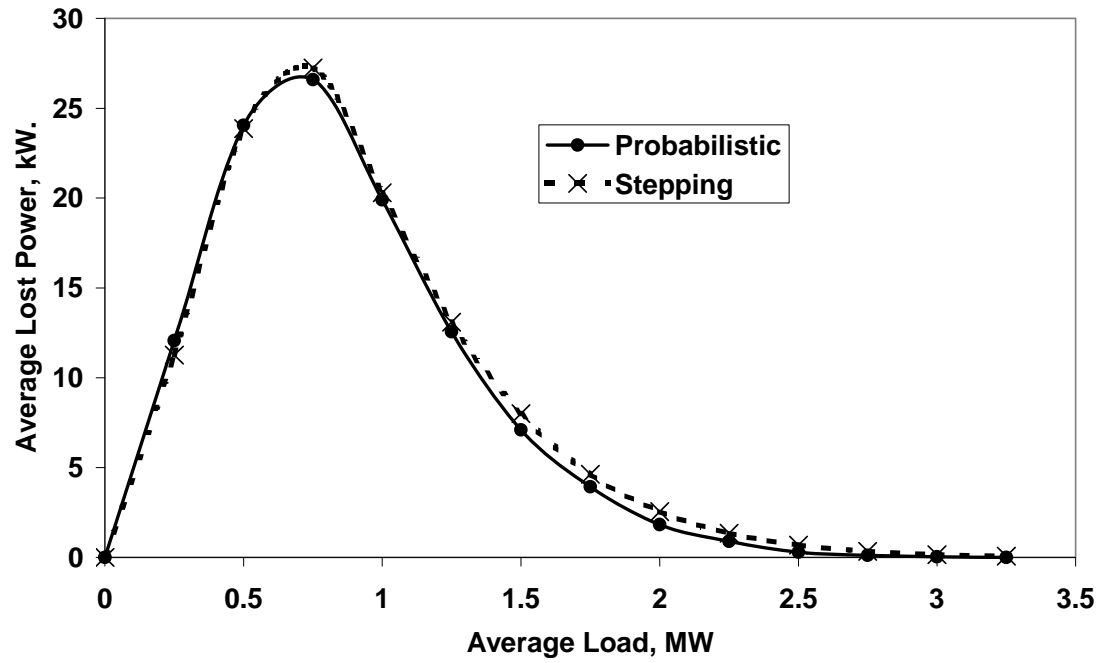

Fig. 10. Average power lost in charging and discharging the store 
The above graphs show that there is a critical average load, between 0.5MW and $0.75 \mathrm{MW}$ at which entering the store is matched by power leaving the store. This is the load at which the store is doing most work and the load at which the store loses most power due to its finite efficiency of 75\%, Fig. 10.

This critical average load is close to the average supply of renewable energy. The average measured solar irradiance at RAL is $118.8 \mathrm{~W} \mathrm{~m}^{-2}$, giving a solar capacity factor of $11.88 \%$ and an average solar power of $237.6 \mathrm{~kW}$ from the $2 \mathrm{~kW}$ of solar capacity. The scaled average wind speed is $8 \mathrm{~m} \mathrm{~s}^{-1}$, which together with the turbine power curve produces a wind capacity factor of $39 \%$ and an average power from a 1MW turbine of $390 \mathrm{~kW}$. The long-term average renewable power is therefore about 630kW. Fig. 9 shows the power that would enter or leave the store if it is assumed to be never full or empty (i.e. assumed to be of infinite size); the intersection of the two curves indicates the point where the average power entering the store is exactly equal to average power leaving the store, and that this is indeed at a load of about $630 \mathrm{~kW}$.

The probabilistic method also calculates the fractions of time that the store is full, empty, filling or emptying. It does so by making simple assumptions about the cyclical nature of charging and discharging, the probability distributions of net power to and from the store. It also makes use of the fact that (excluding large power excursions) renewable power is only curtailed when the store is full and load is only unsatisfied when the store is empty, Figs. 11 to 14. 


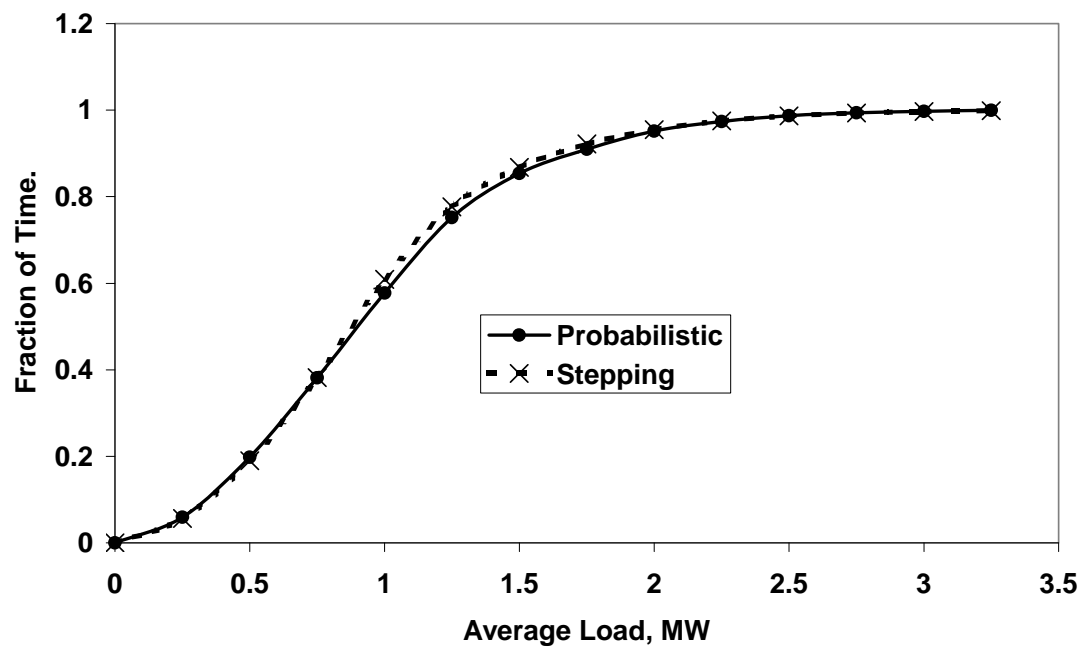

Fig. 11. Fraction of time that the store is empty

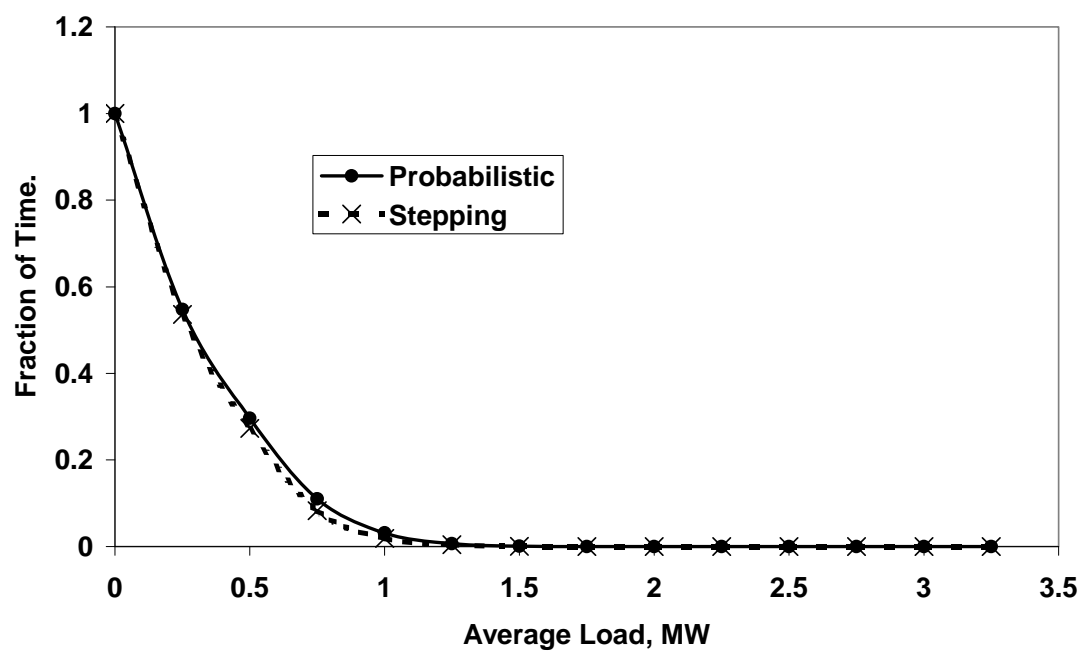

Fig. 12. Fraction of time that the store is full 


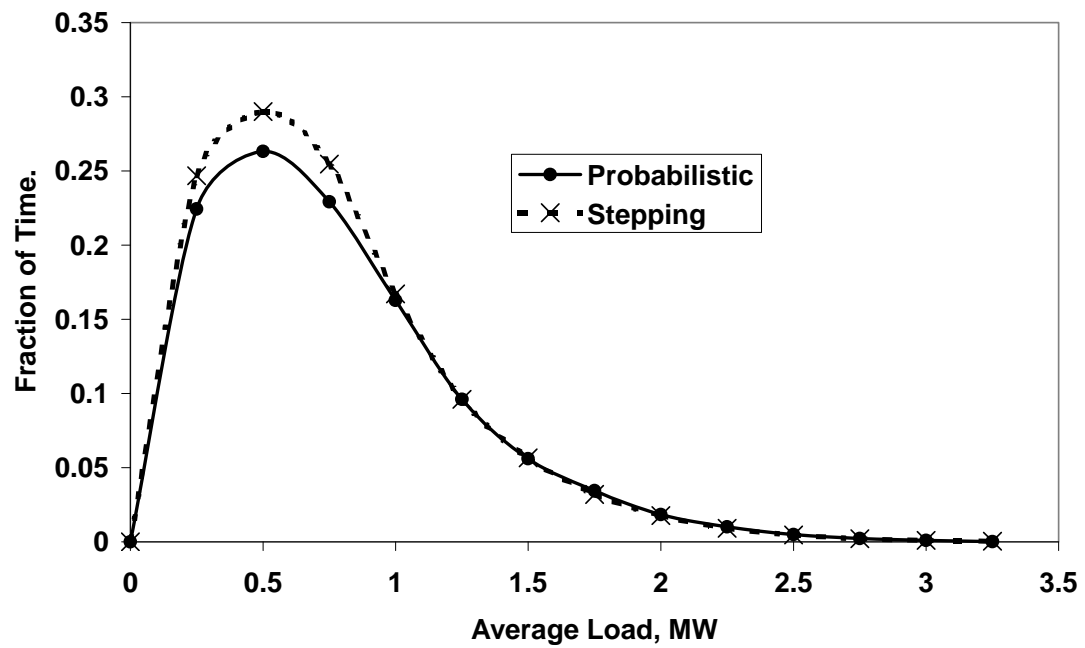

Fig. 13. Fraction of time that the store is emptying

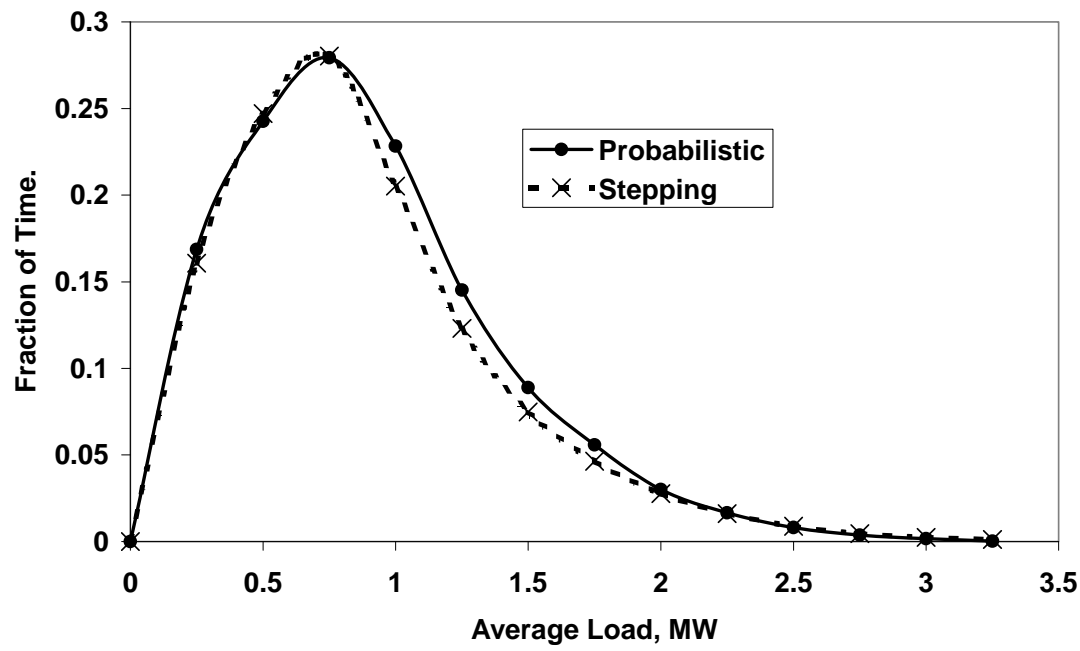

Fig. 14. Fraction of time that the store is filling 
In reality, the power flows to and from the store are limited by the finite energy capacity of the store. The predicted powers supplied to the load, in total and via the store, compare well with the time-step predictions, Fig. 15.

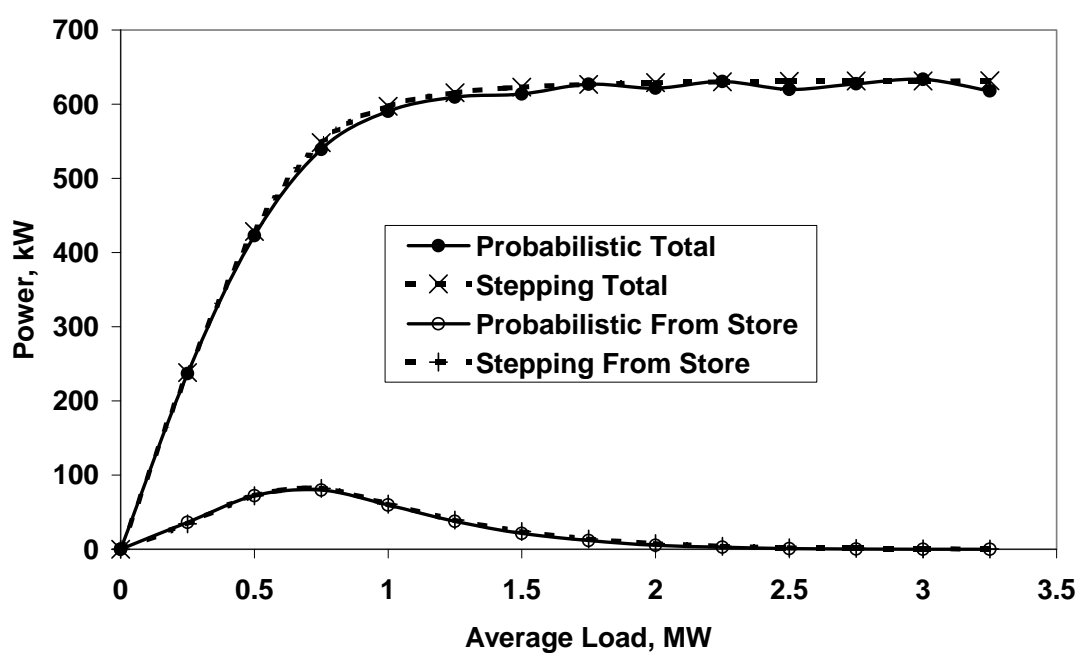

Fig. 15. Load supplied by renewables, total and via the store.

\section{Conclusions}

It is clear from the graphs that the results of the new probabilistic method are in excellent agreement with the time step model. No significant power is lost for average loads above $1 \mathrm{MW}$, despite the 3MW of total renewable capacity. This reflects the fact that the renewable sources are rarely producing their combined rated output and that the bulk of the time-varying surplus is made useful by the storage system. There is however a need for an "auxiliary supply" for all but the lowest loads, but this is easily accommodated by the existing electricity distribution system; indeed it is the normal state of affairs. It is not the intention here to make the local 
load completely independent of external supply, but rather to make better use of the distributed generation.

\section{References}

[1] National Grid Seven Year Statement, 2003. Actual National Grid Summer and Winter Demands for 2002/2003 (Not Weather Corrected), http://www.nationalgrid.com/uk/library/documents/sys 03/dddownloaddisplay.asp?sp =sys Figure 2 3

[2] D. Milborrow "Revolutionary Potential”, Wind Power Monthly, 16(10), October 2000

[3] J.P. Barton and D.G. Infield, "Energy storage and its use with intermittent renewable energy”, IEEE Trans.Energy Convers., 19(2), pp. 441-448, June 2004.

[4] “Windenergie 2000” turbine catalogue, Bundesverband WindEnergie e.V., 2000, ISBN 3-9806657-2-0

[5] John P. Barton and David G. Infield, "Energy Storage and its Use with Wind Power”, IEEE Power Engineering Society General Meeting conference proceedings, $12^{\text {th }}$ to $16^{\text {th }}$ June 2005 , San Francisco, USA, ISBN 0-7803-9157-8, Dispatchable Wind Power Generation, PESGM2005-000168.PDF

[6] U. Hassan and D. M. Sykes, "Wind Structure and Statistics”, in Wind Energy Conversion Systems chapter 2, L. L. Freris, Ed. UK: Prentice Hall, 1990, pp. 11-32. [7] D.G. Infield, "Storage and Logistics”, in Wind Energy Conversion Systems chapter 16, L. L. Freris, Ed. UK: Prentice Hall, 1990, pp. 341-356. 
[8] E.A. Bossanyi, "Comparison of Techniques for Evaluating the Frequency of Wind Turbine Shut-Downs", Proceedings of the $6^{\text {th }}$ BWEA Wind Energy Conference, 1984, UK: Cambridge Univ. Press, pp. 228-238 